\title{
Cognition and Function Research on Fuzzy Anaphora of English and Chinese Narrative Discourse in Computer Science Area
}

\author{
YANG ZhiShang, a \\ ${ }^{1}$ Changsha Medical University, Changsha, Hunan,413000 China \\ a284204813@qq.com
}

Keywords: fuzzy; anaphora; cognition; function

\begin{abstract}
.
This paper makes multi-level analysis and explanation on functions of fuzzy anaphora, it is composed of mutual effect of component factor, associated factor and influence factor, analysis on fuzzy anaphora function involves with 3 aspects such as narrative, pragmatics and rhetoric.
\end{abstract}

\section{Introduction}

With linguistics changes from its attention on separated clause to consideration on actual text, anaphora research begins to pay more attention to semantic, function, pragmatic and cognitive factors beyond of grammar; people's acquaintance on anaphora becomes more and more complete and deep. Scholars both in China and abroad make universal and deep research on anaphora situation in Chinese discourse from many perspectives. Meanwhile, it integrates fuzzy anaphora situation of fuzzy language characteristics but without arousing enough emphasis from researchers. Through measures such as literature analysis, theory research, situation explanation etc, it makes systematic research on fuzzy anaphora situation. Because perpetual motion of objective things have homomorph anaphora with generalized characteristic and zero anaphora is beyond of this research, and then it focuses and analyzes special anaphora.

\section{Analysis on cognition and function of English and Chinese narrative fuzzy anaphora}

Fuzzy anaphora means it is always in the border position of mainstream research, or it is sometimes proposed as one kind of pragmatic failure. In our research, fuzzy anaphora is regarded as one basic part of language fuzziness and one kind of normalized situation. Fuzzy anaphora situation in narrative has different fuzzy degree, which undertakes narrative connection and concept transmission function, meanwhile it displays designated rhetoric functions, it is compared with normal anaphora, corpus quantity is little and distribution is scattered. It collects from many kinds of narrative discourse, making the total sample of summary enough to achieve obvious quantity, and then it makes classification, description and explanation. Demonstration research is the basic work of this research, corpus is mainly chosen from English and Chinese narrative discourse, it will also choose some representative corpus from stylistic style or expository style according to research demand. Secondly, as for the collected corpus, we will make explanation on its production and paraphrasing process based on perspective of cognitive linguistics. Wu will use this classic theory to explain fuzzy anaphora situation, on one hand, it can demonstrate our definition on fuzzy anaphora situation is scientific and feasible or not. One the other hand, it can test applicability of traditional theory in fuzzy anaphora research. The second kind of theory is based on character value theory put forward by Fauconnier and creatively constructs core theory structure focuses on fuzzy anaphora phenomenon explanation. In order to make character value display complete effect in paraphrasing 
mechanism in fuzzy anaphora, we should apply diffusion activation theory and matching theory to make complementary to it. The core theory structure of this research is one integrated structure of using character value as core as well as diffusion and matching theory as complementary. Finally, it makes multi-level analysis and explanation on discourse function of fuzzy anaphora. Fuzzy anaphora is composed of mutual effect of component factor, associated factor and influence factor, anaphora, fuzzy, cognition and discourse function is usually placed in different levels to make research, in view of characteristics of fuzzy anaphora, and we integrate these aspects to make discussion so as to discuss their coordination and restriction relation between them.

\section{A. Evolving anaphora}

Anaphora manes the primary object without being evolved, it constructs direct anaphor, if the referred object corresponds to object evolved, then it corresponds to indirect anaphor, therefore, evolving anaphor means one kind of state between direct anaphor and indirect anaphora.

discourse referent:

text constituents:

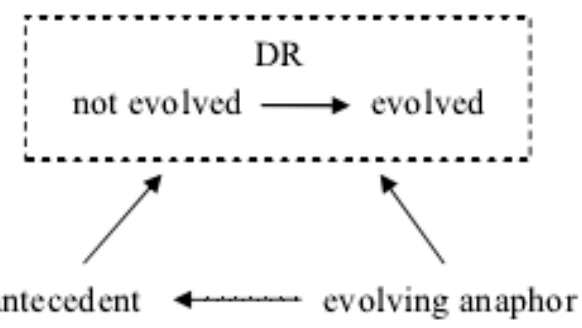

antecedent $\longleftarrow \ldots \ldots-\ldots$ evolving anaphor

Figure 1 Cognition model of field of event

Accessibility marking principle is oriented by association, it aims to make addressee obtain the maximum contextual effect by the minimum treatment cost in the process of understanding accessibility markers. According to accessibility principle, under the same conditions, the short of discourse interval between antecedent and anaphora, the higher of accessibility, the more potential antecedent participates in competition, the low of accessibility, the higher of obvious level of antecedent, the higher of accessibility, the stronger of uniformity between antecedent and anaphor, the higher of accessibility. In fuzzy anaphor situation, fuzzy reference relation constructed between antecedent and anaphor dose not mean addressee will certainly meet extraction difficulty in referent paraphrasing.

Table 1 Accessibility of fuzzy anaphor

\begin{tabular}{|c|c|c|c|c|c|}
\hline category & $\begin{array}{c}\text { Spacing } \\
\text { distance }\end{array}$ & competition & significance & uniformity & accessibility \\
\hline $\begin{array}{c}\text { evolving } \\
\text { anaphor }\end{array}$ & short & low & high,middle & high & Mid-high \\
\hline $\begin{array}{c}\text { Summary } \\
\text { anaphor }\end{array}$ & short & low & low & high & mid-high \\
\hline $\begin{array}{c}\text { indirect } \\
\text { anaphor }\end{array}$ & $\begin{array}{c}\text { long or } \\
\text { short }\end{array}$ & high & low & high & mid-low \\
\hline $\begin{array}{c}\text { ambiguous } \\
\text { anaphora }\end{array}$ & $\begin{array}{c}\text { Long or } \\
\text { short }\end{array}$ & high & low & high & mid-low \\
\hline
\end{tabular}

Accessibility theory can not only be applied in analyzing and paraphrasing, it is also suitable to analyze fuzzy anaphor. Here, we just use 4 parameters in accessibility theory to make some rough analysis on fuzzy anaphor, which needs to make demonstration research based on large-scale corpus and make further demonstration.

\section{B. Cognitive reference theory}

Human have one basic cognition ability, which is we can construct psychological contact of the other entity concept through one entity concept; this is anaphor reference point model. The basic assumption of this theory is as follows: because feature degree of every perceived entity is different, some entities are easy to be positioned compared with other entities, these entities are more obvious as for observers, while perceiving other entities needs to take great effort. These obvious entities are the reference $\mathrm{R}$ of people on knowing this world; people perceive and know other entities through 
reference point, which is target $\mathrm{T}$ of cognition activity. One cognition reference point can activate one related psychological space, which is domain $\mathrm{D}$ of this reference point. In this field, we can perceive more possible targets; the real cognitive target is $\mathrm{T}$, which is indicated by figure 2 :

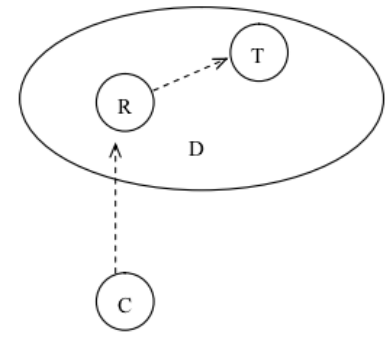

C cognition object $\mathrm{D}$ domain $\mathrm{R}$ referent $\mathrm{T}$ target $\cdots \cdots \cdot$. psychological route

Figure 2 Langacker cognition reference point model

\section{Evolving anaphora}

Evolving anaphora means the kind has the lightest fuzzy degree and easiest cognitive processing. The antecedent in discourse, which is reference point R1, it activates component related to R1 in its domain $\mathrm{D}$, of which the most obvious is cognitive target $\mathrm{T}^{\prime} 1$, it is regarded as the next cognition reference point R'2 in the evolving process, T'2 undertakes the next cognition reference point R' 3 in the evolving process, $R^{\prime} 3$ consequently activates the next new cognition target R'4... ..., until to the final target Rno R1,R' 2 , R' $3 \ldots$. . . Rn is regarded as the advance item of the detailed stage in the discourse development process. Through gradual evolution, partial characteristics change but object has no change, when it involves to Rn, it activates the final target $\mathrm{T}$. Therefore, cognitive object c can transmit concept through reference point R1 (antecedent) in discourse and undergoes interlocked chain evolving, and finally completes establishment of the whole anaphora relation through target $\mathrm{T}$ (anaphora). In the process of evolving paraphrasing, once addressee confirms cognitive target through reference point, the previous reference point may retreat from the present topic domain, the activated cognitive target is distinctively understood as reference point of new target, which will promote more and more target to be understood one after another, so that it promotes development of discourse, which is indicated by figure 3 :

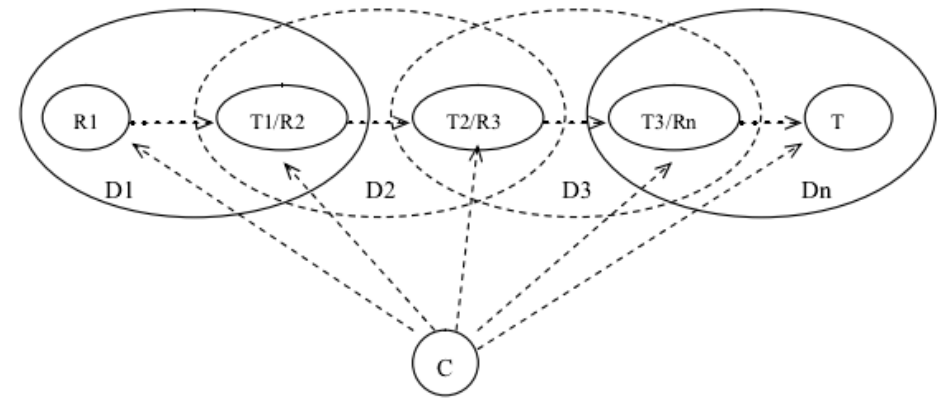

C cognition object $\mathrm{D}$ domain $\mathrm{R}$ reference point $\mathrm{T}$ target $\cdots \cdots{ }_{\text {psychological route }}$

Figure 3 Cognition reference point model of evolving anaphora

In overview anaphora, cognition object extracts and summarizes common nature many antecedents, which is reference point $\mathrm{R}^{\prime} 1, \mathrm{R}^{\prime} 2, \mathrm{R}^{\prime} 3$. . ...extracts and summarizes as core concept reference point $R$, while respectively activated domain $D^{\prime} 1, D^{\prime} 2, D^{\prime} 3 \ldots .$. of $R^{\prime} 1, R^{\prime} 2$, $R^{\prime}$ 3 . ....overlaps and mutually integrates, which forms the final domain $\mathrm{D}$ includes many domain common nature, in this domain $\mathrm{D}$, cognition object $\mathrm{C}$ determines final cognition target $\mathrm{T}$ through reference point $\mathrm{R}$ to complete concept anaphora, which is indicated by figure 4 : 


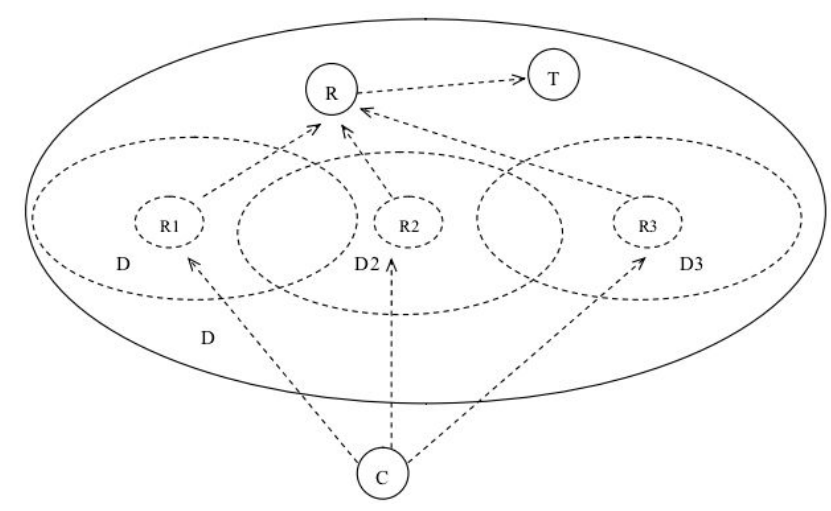

$\mathrm{C}$ cognition object $\mathrm{D}$ domain $\mathrm{R}$ reference point $\mathrm{T}$ target $\cdots \cdots{ }_{\text {psychological route }}$

Figure 4 Cognition reference point of conceptual anaphora

Indirect anaphora means phenomenon as for the above-mentioned 2 kinds anaphora phenomenon, fuzzy degree is high and cognition process is complicated. Cognition object firstly activates the first reference point R', R, through antecedent. In other domain $\mathrm{D}$, through regular relation, such as one part of integral, entity-function nature, action-result place, hyponymy etc, it activates cognition target $\mathrm{T}^{\prime}$, cognition target $\mathrm{T}$ should be used as actual cognition reference point $\mathrm{R}$, in its activated domain $\mathrm{D}$, it activates final cognition target $\mathrm{T}$, construction relation, which is indicated by figure 5 :

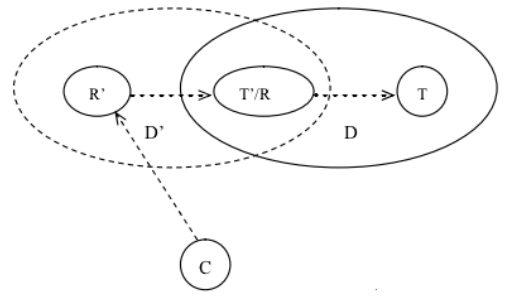

$\mathrm{C}$ cognition object $\mathrm{D}$ domain $\mathrm{R}$ reference point $\mathrm{T}$ target $\cdots \cdots{ }_{\text {psychological route }}$

Figure 5 Reference point model of indirect anaphora cognition

D Syntactic ambiguity Anaphora

The obvious distinction between syntactic ambiguity anaphora and concept anaphor lies in as follows: according to characteristics of human cognate the world, an Hoek C 1997: 58-59 thinks that factors of affecting choice of reference point is as follows: the first is salience, if $\mathrm{X}$ is included in context of $\mathrm{Y}$, it is more outstanding, then $\mathrm{Y}$ is possible to be chosen as reference point. The second is $\mathrm{C}$ linear word order, under condition of the same conditions, if $\mathrm{X}$ is perceived earlier than $\mathrm{Y}, \mathrm{X}$ is more likely to be chosen by reference point. The third is concept association, which is the close degree of concept semantic relation between 2 nominal elements; this is determined by participated structure relation of 2. Cognition object chooses linear relations in several antecedents.

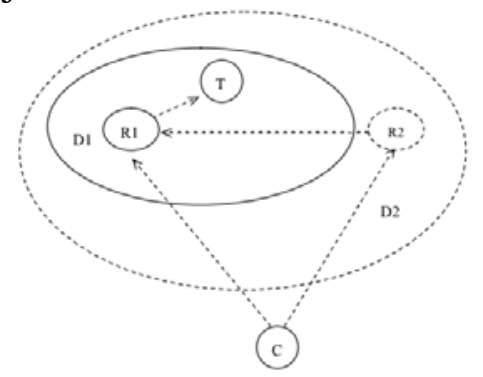

Figure 6 Reference point model of indirect anaphor

Conceptual matching means the consistent relation between some components or all components of 2 concepts; it is the fundamental base to establish meaning relevance among concept. Any 2 concept, only if they have meaningful relevance between them, they must have component with co-core, the process of extracting co-core component between them is matching. Figure 7 presents the complete matching condition of 2 concepts. 


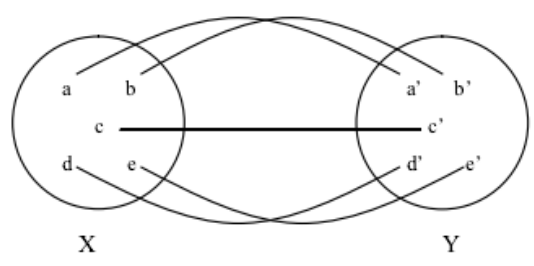

Figure 7 Complete marching

\section{Conclusion}

Cognition structure can be simple or complicated, this research just makes discussion on it from micro, middle and macro level of discourse, but content involved by micro, middle and macro is not endless, of course, it is not all the content of discourse consistent research. Language and thinking complementary to each other simultaneously, cognition structure and language can not be separated, all has dynamic nature. Learning discourse must transfer structure knowledge, so cognition structure can be used as one kind of method or strategy of discourse analysis.

\section{References}

[1]Zhou Hong. Narrative Discourse Research Comment of China and Abroad in Recent 20 Years [J]. Modern Chinese (language research vision), 2017, 04:4-8+2.

[2]Wang Jie. Research on Fuzzy Anaphor Paraphrasing Mechanism Based on Cognition Reference Point [J]. Journal of Suzhou University of Science and Technology (social science edition), 2016, 02:89-96.

[3]Xun Tianyun. Transformation Strategy of Traditional Name in Discourse [J]. Journal of ZhaoQing University, 2016, 03:39-44.

[4]Wang Jie. Research on Fuzzy Anaphor Phenomenon Paraphrasing Based on Relationship between Lattice Frames [J]. Journal of Suzhou University of Science and Technology (social science edition), 2014, 04:86-91.

[5]Yang Youwen, Yin Rui. Comparative Research on Category Pattern and Its Example in English and Chinese Discourse [J]. Journal of Beijing International Studies University, 2015, 06:1-12. 